\title{
Influência da hidrocinesioterapia no equilíbrio postural de idosas institucionalizadas
}

\author{
Estele Caroline Welter Meereis ${ }^{1}$ \\ Camile Favretto ${ }^{1}$ \\ Jaqueline de Souza ${ }^{2}$ \\ Marisa Pereira Gonçalves ${ }^{1}$ \\ Carlos Bolli Mota ${ }^{3}$ \\ ${ }^{1}$ Departamento de Fisioterapia e Reabilitação, Centro de Ciências da Saúde, Universidade \\ Federal de Santa Maria, RS, Brasil \\ ${ }^{2}$ Departamento de Fisioterapia, Centro de Ciências da Saúde, Universidade Federal do Pampa, \\ Uruguaiana, RS, Brasil \\ ${ }^{3}$ Departamento de Métodos e Técnicas Desportivas, Centro de Educação Física e Desportos, \\ Universidade Federal de Santa Maria, RS, Brasil
}

\begin{abstract}
Resumo: O objetivo deste estudo foi avaliar a influência de um programa de hidrocinesioterapia em piscina rasa no equilíbrio postural de idosas institucionalizadas. Participaram do estudo 8 idosas institucionalizadas com média de idade de $62,95 \pm 2,87$ anos. O equilíbrio postural foi avaliado por meio de uma plataforma de força. Foram realizadas 3 tentativas em cada condição do teste de organização sensorial (TOS) previamente e posteriormente a intervenção de hidrocinesioterapia em piscina rasa. As variáveis analisadas foram: amplitude de deslocamento do centro de pressão (COP) na direção ântero-posterior, médio-lateral e velocidade de deslocamento do COP. Foi aplicado o teste Wilcoxon para a comparação entre as avaliações pré e pós a intervenção. Foi observada uma melhora no controle postural após a intervenção, visto que ocorreu diminuição do deslocamento do centro de pressão, principalmente na direção ântero-posterior. Para o grupo de idosas institucionalizadas participantes deste estudo, a hidrocinesioterapia realizada em piscina rasa foi capaz de melhorar o equilíbrio postural.
\end{abstract}

Palavras-chave: Equilíbrio postural. Hidroterapia. Idoso. Instituição de longa permanência para idosos.

\section{Influence of hydrokinetic therapy in postural balance of institutionalized elderly people}

\begin{abstract}
The purpose of the present study was to evaluate the influence that a hydrokinetic therapy program in shallow water has on postural balance of institutionalized elderly women. In this study 8 institutionalized elderly, with a mean age of $62.95 \pm 2.87$ years participated. The postural balance was evaluated through the force platform. 3 attempts in each sensory organization test (SOT) previously and subsequently the intervention of hydrokinetic therapy in shallow water were made. The variables analyzed were: displacement amplitude of the pressure center (COP) in the anteroposterior, mediolateral direction and displacement speed of the COP. An improvement in postural control after the intervention was observed, where a decrease on the displacement of the pressure center happened, especially in the anteroposterior direction. For the group of institutionalized elderly participants in this study, hydrokinetic therapy performed in shallow water was able to improve postural balance.
\end{abstract}

Keywords: Postural balance. Hydrotherapy. Aged. Homes for the Aged.

\section{Introdução}

O controle do equilíbrio requer a manutenção do centro de gravidade sobre a base de sustentação durante situações estáticas e dinâmicas (DANIEL et al., 2010). Para o controle do equilíbrio corporal são necessárias contribuições dos sistemas visual, vestibular e proprioceptivo (BELLEW et al., 2009).

Com o envelhecimento, esses sistemas são afetados e várias etapas do controle postural podem ser suprimidas, aumentando o risco de quedas (FERREIRA, 2003). As quedas se tornaram um fator de grande relevância epidemiológica na população idosa; Greve et al. (2007) afirmam que a queda é o tipo de acidente mais frequente no idoso e suas complicações, a principal causa de morte naqueles com mais de 65 anos.

Henry et al. (2001) citam que os níveis de inatividade física nesta população são elevados, os quais são mais acentuados nos idosos institucionalizados. Adicionalmente a isso, o medo 
de cair em idosos institucionalizados é bastante frequente sendo considerado como um fator de risco para a independência do indivíduo (BROUWER et al., 2003). Diante disso, observase que é importante a investigação de formas alternativas buscando a prevenção das quedas em idosos.

A hidrocinesioterapia é um recurso fisioterapêutico que busca a reabilitação humana através dos princípios da aplicação de exercícios físicos aliado às propriedades físicas da água. Essas propriedades físicas podem agir de maneira a aumentar a amplitude de movimento, a flexibilidade e a força muscular. Assim, o uso do meio aquático poderia ser um coadjuvante terapêutico para melhora do equilíbrio corporal e prevenção de quedas em idosos (RESENDE et al., 2008). Alguns estudos vêm investigando a ação da fisioterapia aquática no equilíbrio e manutenção da postura em idosos (RESENDE et al., 2008; CANDELORO e CAROMANO, 2007). Esses estudos utilizaram questionários para fins de avaliação e apontam para uma melhora no equilíbrio postural. Entretanto, há questionamentos em relação à influência deste tratamento em condições que alguns dos sistemas sensoriais responsáveis pelo equilíbrio sejam suprimidos.

De acordo com Ronda et al. (2002), as condições do teste de organização sensorial (TOS) permitem analisar a contribuição dos sistemas sensoriais no controle postural. As medidas de deslocamento do centro de pressão (COP) durante posição ortostática são resultados do controle neuromuscular que o corpo efetua para manter o controle postural (STEL et al., 2003; MOCHISUKI; AMADIO, 2003). Portanto, ao avaliar-se os deslocamentos do COP paralelamente às situações propostas pelo TOS é possível inferir a respeito da contribuição dos sistemas sensoriais sobre a manutenção do controle postural. Diante disso, o objetivo deste estudo foi avaliar a influência de uma proposta de intervenção hidrocinesioterapêutica em piscina rasa no controle postural de idosas institucionalizadas, bem como, sua influência sobre os sistemas sensoriais envolvidos na manutenção e controle do equilíbrio.

\section{Metodologia}

A pesquisa realizada foi aprovada pelo Comitê de Ética e Pesquisa da Universidade Federal de Santa Maria (CAAE - 010.0.243.000-08 2008), conforme as normas estabelecidas na Resolução 196/96 do Conselho Nacional de Saúde sobre pesquisa envolvendo seres humanos.

Foram utilizados os seguintes critérios de inclusão para seleção dos sujeitos: gênero feminino, idade superior a 60 anos e disponibilidade de tempo para realização das atividades propostas. A opção por mulheres visou maior homogeneidade da amostra. Os critérios de exclusão foram determinados segundo as principais contra-indicações para a prática de atividades aquáticas, tais como: febre, erupção cutânea contagiosa, doença infecciosa, doença cardiovascular grave, história de convulsões não controladas, hipotensão ou hipertensão grave e incontinência urinária (BIASOLI; MACHADO, 2006). Também foi fator de exclusão a incapacidade para manutenção da postura em pé e marcha, a qual se torna necessária para a realização dos testes. O grupo de estudo foi composto por oito idosas do gênero feminino, das quais foram mensuradas as medidas de massa e estatura corporal.

Todas as participantes realizaram exame médico previamente ao início do estudo. As atividades aquáticas foram realizadas em grupo sob o acompanhamento de monitor (um monitor para cada idosa), em piscina rasa $(80 \mathrm{~cm}$ de profundidade) uma vez por semana, totalizando 15 sessões, cada uma com duração de 50 minutos. O protocolo de hidrocinesioterapia foi adaptado dos modelos de Resende et al.(2008) e Campion (2002) sendo composto por exercícios físicos para melhorar a coordenação motora, o equilíbrio, a força muscular, a resistência, a respiração, além de adaptação ao meio líquido e atividades lúdicas e de recreação para proporcionar as idosas um momento de lazer e descontração.

Para a aquisição dos dados referentes ao equilíbrio corporal foi utilizada uma plataforma de força (OR6-6, Advanced Mechanical Tecnology Incorporation, USA). A avaliação da contribuição relativa dos sistemas responsáveis pelo equilíbrio (proprioceptivo, visual e vestibular) foram avaliados através do TOS utilizando-se a posturografia dinâmica validada por Castagno (1994), assim como estudo de Teixeira et al. (2011). Para a realização do TOS os indivíduos foram instruídos a permanecer o mais estático possível, sob a plataforma de força dentro de uma cabine de $1 \mathrm{~m}^{2}$, com altura de $2 \mathrm{~m}$, composta de listras horizontais, claras e escuras de $10 \mathrm{~cm}$ cada 
uma. A cabine é um sistema mecânico simples e pode mover-se $20^{\circ}$ para frente e para trás. Além disso, foi utilizada uma almofada de $10 \mathrm{~cm}$ de espessura, de $50 \mathrm{~cm} \times 50 \mathrm{~cm}$, sob os pés do indivíduo, ou seja, entre os pés e a plataforma de força visando à manipulação do sistema sensorial. A seguir serão descritas as condições testadas pelo TOS:

TOS I - avalia o sistema visual, o proprioceptivo e o vestibular. Indivíduo com olhos abertos e fixos na horizontal, plataforma de apoio fixa e cabine visual fixa durante 20 segundos.

TOS II - avalia os sistemas proprioceptivos e vestibulares. Olhos fechados, plataforma de apoio fixa.

TOS III - avalia o sistema proprioceptivo, vestibular e, sobretudo, o visual. Olhos abertos, cabine visual oscilante, sendo 10 segundos com cabine inclinando-se até $20^{\circ}$ para frente e 10 segundos voltando a posição inicial, em uma velocidade média de $2 \%$ seg. Plataforma de apoio fixa.

TOS IV - avalia principalmente o sistema proprioceptivo. Visão fixa no horizonte e a cabine visual fixa durante os 20 segundos de teste. Base de apoio é oscilante (almofada de $10 \mathrm{~cm}$ de densidade entre os pés do indivíduo e o solo).

TOS V - avalia os sistemas vestibular e proprioceptivo, oferecendo maior dificuldade devido aos olhos estarem fechados, e plataforma de apoio oscilante em uma velocidade média de $2 \%$ seg.

TOS VI - avalia os sistemas proprioceptivo, visual e vestibular. Este teste requer a visão normal com cabine visual oscilante, assim como a base de apoio, devido à presença da almofada.

Para a coleta de dados todos os indivíduos se mantiveram com os pés descalços separados na largura do quadril. Além disso, a plataforma de força foi marcada na primeira tentativa de cada indivíduo para que o posicionamento dos membros inferiores fosse repetido em todas as condições. Foram realizadas três tentativas de 30 segundos cada participante.

A frequência utilizada para aquisição dos dados da plataforma de força (forças e momentos) foi de $100 \mathrm{~Hz}$. O deslocamento do centro de força ao longo do tempo resulta numa trajetória cuja qual está relacionada ao movimento do centro de massa e, portanto, indiretamente relaciona-se com 0 controle postural. As seguintes variáveis derivadas da aferição do centro de pressão foram analisadas: amplitude do deslocamento do COP na direção ântero-posterior (COPap), amplitude do deslocamento do COP na direção médio-lateral (COPml) e velocidade média de deslocamento do COP (COPvel). Os dados brutos provenientes da plataforma foram filtrados por um filtro passa-baixa Butterworth, de 4ª ordem na frequência de $10 \mathrm{~Hz}$, eliminando assim, possíveis interferências do ambiente.

Os diferentes parâmetros de deslocamento do COP contêm informações diferentes sobre 0 controle de postura, sendo que, segundo a literatura os deslocamentos médio-lateral e ântero-posterior são bons preditores de risco de quedas, em especial o primeiro; a velocidade do COP é referenciada como importante parâmetro na comparação entre situações avaliadas (RAYMAKERS et al., 2005).

\section{Resultados}

Participaram da pesquisa oito (8) indivíduos do gênero feminino cujas características do grupo estão ilustradas na tabela 1.

Tabela 1. Características do grupo estudado.

\begin{tabular}{|c|c|c|}
\hline $\mathrm{n}=8$ & Média \pm DP & Mediana (p25-p45) \\
\hline Idade (anos) & $62,95 \pm 2,87$ & $60,5(60-62,75)$ \\
\hline Massa (kg) & $75,34 \pm 7,55$ & $73,55(70,55-79,85)$ \\
\hline Estatura (m) & $1,54 \pm 0,15$ & $1,54(1,52-1,55)$ \\
\hline IMC $\left(\mathrm{kg} / \mathrm{m}^{2}\right)$ & $31,50 \pm 3,63$ & $30,50(29,25-33,5)$ \\
\hline
\end{tabular}

Para a análise estatística foi utilizado o Software SPSS (Statistical Package for the Social Science) versão 13.0 para Windows. Foi aplicado o teste de Shapiro-Wilk para avaliação da distribuição dos dados. Como os dados não apresentaram distribuição normal, foi utilizado o teste Wilcoxon para comparar o equilíbrio postural pré e pós intervenção e ANOVA de Friedman para comparação das variáveis relacionadas aos sistemas envolvidos na manutenção do equilíbrio em cada situação. O nível de significância adotado em todos os testes foi de $5 \%(p<0.05)$. 
Os resultados referentes aos parâmetros avaliados no estudo estão descritos na Tabela 2. Os deslocamentos do COP na direção ânteroposterior (ap) mostraram uma redução estatisticamente significativa nas condições avaliadas nos TOS II, III, IV, V e VI, apontando para uma melhora no controle postural dos participantes após a prática da hidrocinesioterapia.
O deslocamento do COP na direção médiolateral $(\mathrm{ml})$ apresentou menores diferenças dentre as condições avaliadas, em comparação ao deslocamento do COP na direção ânteroposterior. Foram encontradas reduções significativas nos deslocamentos do COP (ml) apenas nas situações dos TOS II e III. Em relação ao parâmetro de velocidade de oscilação do COP, houve redução significativa apenas na condição TOS III.

Tabela 2. Dados referentes à avaliação do equilíbrio postural nas seis condições sensoriais (TOS)

\begin{tabular}{|c|c|c|c|c|c|}
\hline & & & Pré & Pós & \\
\hline & & & Mediana (p25-p45) & Mediana (p25-p45) & Wilcoxon \\
\hline \multirow{3}{*}{ TOS I } & $\overline{\bar{S}}$ & COPap & $2,39(2,13-3,02)$ & $2,07(1,69-2,76)$ & 0,11 \\
\hline & (\$) & COPml & $1,76(1,47-1,99)^{\mathrm{ab}}$ & $0,87(0,78-0,99)^{A}$ & 0,23 \\
\hline & & COPvel & $1,24(1,14-1,43)$ & $1,12(0,90-1,23)$ & 0,40 \\
\hline \multirow{3}{*}{ TOS II } & & COPap & $2,30(1,96-2,92)$ & $2,11(1,95-2,52)$ & $0,00^{*}$ \\
\hline & (8) & COPml & $1,53(0,82-1,73)^{\mathrm{ab}}$ & $1,30(1,07-1,60)^{A B}$ & $0,00^{*}$ \\
\hline & 1 & COPvel & $1,32(1,17-1,54)$ & $1,29(1,08-1,71)$ & 0,67 \\
\hline \multirow{3}{*}{ TOS III } & & COPap & $3,66(2,87-4,25)$ & $3,53(2,89-4,13) 5$ & $0,00^{*}$ \\
\hline & & COPml & $2,29(1,28-2,77)^{a}$ & $1,18(1,09-1,42)^{A B}$ & $0,01^{*}$ \\
\hline & & COPvel & $1,68(1,38-2,17)$ & $1,47(1,40-1,71)$ & $0,01^{*}$ \\
\hline \multirow{3}{*}{ TOS IV } & & COPap & $2,69(2,36-3,42)$ & $2,58(2,08-3,02)$ & $0,04^{*}$ \\
\hline & 16 & COPml & $2,05(1,01-2,59)^{a b}$ & $1,67(1,24-1,95)^{A B}$ & 0,55 \\
\hline & & COPvel & $1,51(1,32-1,79)$ & $1,49(1,29-1,77)$ & 0,87 \\
\hline \multirow{3}{*}{ TOS V } & & COPap & $4,50(3,77-5,91)$ & $3,56(3,05-4,25)$ & $0,00^{*}$ \\
\hline & $\mathbb{N}$ & COPml & $2,38(1,81-2,77)^{a b}$ & $2,34(1,83-3,68)^{A B}$ & 0,67 \\
\hline & 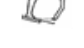 & COPvel & $2,36(1,84-2,60)$ & $2,35(1,79-2,31)$ & 0,71 \\
\hline \multirow{3}{*}{ TOS VI } & & $\mathrm{COPap}^{\mathrm{C}}$ & $5,78(4,91-7,73)$ & $5,12(4,99-7,23)$ & $0,00^{*}$ \\
\hline & & COPml & $3,08(2,18-3,68)^{b}$ & $2,74(2,17-3,88)^{B}$ & 0,09 \\
\hline & & COPvel & $2,69(1,74-3,59)$ & $2,52(1,34-4,14)$ & 0,98 \\
\hline
\end{tabular}

Legenda: COPap: deslocamento do centro de pressão na direção ântero-posterior; COPml: deslocamento do centro de pressão na direção médio-lateral, COPvel: Velocidade Média do deslocamento do COP. P 25-75: Percentil de $25 \%$ e $75 \%$ de distribuição. ${ }^{\text {ab }}$ : Letras minúsculas diferentes para mesma coluna indicam diferença estatisticamente significante $(p<0,05)$ para $C O P m l$ no pré teste. ${ }^{A B}$ : Letras maiúsculas para mesma coluna indicam diferença estatisticamente significante $(p<0,05)$ para COPml no pós teste.

Fonte: Dados da pesquisa.

Também se observa na Tabela 2 as diferenças entre as condições de TOS analisadas antes e após o tratamento. A ANOVA revelou que no período pré-tratamento havia diferença estatisticamente significativa entre as condições TOS III e TOS VI no deslocamento médio lateral (COPml), sendo que no TOS $\mathrm{VI}$, no qual o sistema proprioceptivo encontra-se perturbado as 
idosas apresentaram maiores amplitudes de deslocamento do COP. Já no período póstratamento ocorreu diferença entre os TOS I e TOS VI em relação ao referido deslocamento. $\mathrm{Na}$ condição de TOS I, todos os sistemas sensoriais encontram-se disponíveis, portanto é esperado que nesta condição aconteça um melhor controle postural, como ocorreu na avaliação póstratamento.

\section{Discussão}

A proposta deste estudo foi avaliar a influência da hidrocinesioterapia no equilíbrio postural de idosas institucionalizadas. Os resultados desta pesquisa corroboram com os estudos de De Vito et al. (2003) e Liu-Ambrose et al. (2004) os quais também observaram uma melhora no equilíbrio após tratamento com atividade física.

De forma geral, pode-se observar que na maioria das situações a amplitude do deslocamento do COPap foi significativamente maior na avaliação pré-tratamento. Nesta direção, a manutenção do controle postural depende predominantemente da articulação do tornozelo, portanto pode-se inferir, que na avaliação prévia ao tratamento as idosas apresentavam uma capacidade diminuída dos músculos do tornozelo em produzir ajustes neuromusculares para mantê-las na postura em pé, possivelmente pela redução de informações proprioceptivas disponíveis, provenientes da região distal dos membros inferiores ou ainda por uma maior perda de unidades motoras em músculos distais dos membros inferiores comuns ao envelhecimento (AMIRIDIS et al., 2003). Na avaliação póstratamento observou-se uma redução nesse deslocamento que aponta para uma melhora no fortalecimento e ativação dos músculos desta articulação, sugerindo uma influência positiva da hidrocinesioterapia para a melhora destas funções.

$\mathrm{Na}$ condição do TOS II ocorreram diferenças estatisticamente significativas entre as avaliações pré e pós tratamento, observadas em ambos os deslocamentos do COP (ântero-posterior e médio-lateral). Nessa condição a informação visual é suprimida, ou seja, os sistemas vestibular e proprioceptivo estão informando aos centros superiores sobre a postura e, assim, estão sendo o foco dessa avaliação. A redução observada neste parâmetro e condição aponta para uma influência positiva do tratamento proposto sobre esses sistemas.
De forma semelhante, na condição TOS III os sistemas vestibular e proprioceptivo são avaliados, no entanto nessa situação a informação visual é manipulada, dificultando a manutenção do equilíbrio em virtude das informações sensoriais divergentes. Nessa condição houve diferença nas três variáveis analisadas, com reduções significativas quando comparadas às condições do pré-tratamento. $O$ presente resultado informa que mesmo diante de uma informação sensorial distorcida (visão) os demais sistemas sensoriais foram capazes de responder adequadamente refletindo em menores deslocamentos e, consequentemente, menores riscos de instabilidades e quedas.

Segundo Resende et al. (2008), a terapia aquática é uma forma de melhorar a condição de equilíbrio, pois favorece o aprimoramento das condições de recepção de informações sensoriais, em especial ativando os músculos antigravitacionais para reestruturação da postura e manutenção do equilíbrio. Adicionalmente a isso, Ricci et al. (2009) referem que as condições de dificuldade que o idoso é repetidamente exposto durante 0 tratamento podem gerar aprendizado da tarefa, assim como também é possível treinar a confiança nos demais sistemas e diminuir a dependência em um único sistema sensorial.

No TOS IV em que o sistema proprioceptivo é avaliado houve redução do deslocamento do COPap na avaliação pós-tratamento. Nessa condição os indivíduos são obrigados a responder a instabilidade oferecida pela superfície que se encontra instável, que segundo a literatura, aumenta a dificuldade da tarefa (RAY et al., 2008). Em vista disso, os resultados sugerem que o tratamento proposto pode ter estimulado o sistema proprioceptivo de maneira a facilitar os ajustes e a força muscular para adequar o controle postural à condição, como sugere Resende et al. (2008).

Semelhantes mudanças foram encontradas com reduções de deslocamento nas condições do TOS V e VI na qual o principal sistema avaliado é o vestibular. Baloh et al. (1992) indicam que o TOS $V$ avalia a informação referente ao sistema vestibular, uma vez que o visual não esta em ação e 0 proprioceptivo está alterado, similarmente à situação do TOS VI, na qual o sistema proprioceptivo encontra-se alterado e o sistema visual é manipulado. Isso é indicativo de 
que o controle exercido pelo sistema vestibular sobre 0 equilíbrio, representado pelo deslocamento na direção ântero-posterior pode ser treinado e melhorado com a realização de exercícios de hidrocinesioterapia, visto que o ambiente aquático proporciona um aumento da confiança do indivíduo e reduz o medo de cair devido à flutuação, o que permite uma maior movimentação do indivíduo além de seus limites de estabilidade, sem temer as conseqüências de queda que podem ocorrer no solo Resende et al. (2008).

Quando comparados os valores das condições TOS entre si no pré-tratamento e pós-tratamento, foram observadas diferenças apenas no deslocamento do COP na direção médio-lateral para algumas condições, já na direção ânteroposterior não ocorreram diferenças estatísticas. Todavia, segundo Raymakers et al., (2005) os deslocamentos médio-lateral são os melhores preditores de quedas e, por isso, os resultados em que se esperam maiores reduções de deslocamento para confirmar o efeito positivo do tratamento proposto. Sendo que os deslocamentos ântero-posteriores envolvem um número maior de articulações para manutenção do equilíbrio nessa direção (articulação do tornozelo e quadril), já as oscilações na direção médio-lateral são provenientes apenas da articulação do quadril em função da anatomia do tornozelo, refletindo em maior expressão dos ajustes posturais (MOCHIZUKI et al., 2006).

No pré-tratamento as diferenças do COP (médio-lateral) ocorreram entre as condições do TOS III e IV, que comparam a presença ou não de instabilidade proprioceptiva mediante informação visual distorcida. Previamente ao tratamento a associação dessas duas condições que dificultam a manutenção postural mostrou diferenças com maiores deslocamentos, apontando para uma maior dificuldade em garantir a manutenção do equilíbrio. Porém, quando essas condições foram novamente comparadas após o tratamento, não houve diferenças significativas, mostrando que houve um melhor ajuste e estabilização da postura em situações de instabilidade. Gauchard et al. (2003) explicam que em condições normais a propriocepção e a informação sensorial da superfície cutâneo plantar são fontes importantes para manutenção do controle postural, entretanto são diante das situações de instabilidades, como alteração da visão (escuro ou alterações na acuidade visual) e pisos instáveis, que há maiores propensões dos idosos sofrerem com desequilíbrios e risco de quedas. A hidrocinesioterapia através da possibilidade ampliada em trabalhar o equilíbrio em diferentes posturas (RESENDE et al. 2008; CAMPION, 2000), inclusive nas mais instáveis, pode ter permitido o treino dos sistemas sensoriais para agirem mais efetivamente nas circunstâncias de instabilidade postural.

A comparação entre TOS I e VI, não apresentou diferenças previamente ao tratamento, mas no pós-tratamento houve um aumento no deslocamento do COPml. $\mathrm{Na}$ condição do TOS I todos os sistemas sensoriais estão agindo com informações e, na condição do TOS VI os sistemas visual e proprioceptivo estão sendo perturbados. Embora o presente resultado não tenha sido o esperado, na literatura outros estudos como de Wolfson et al. (1992) e Teixeira et al (2011) também referem um aumento de oscilação quando a visão e propriocepção são alteradas (condição VI).

Os resultados deste estudo sugerem uma melhora no controle da postura de idosas institucionalizadas após a prática da hidrocinesioterapia, corroborando com o estudo de Carvalho et a. (2007) onde o equilíbrio de idosos institucionalizados praticantes de atividades físicas (treino de resistência aeróbia, força muscular, equilíbrio, flexibilidade e coordenação) mostrou-se melhor comparativamente aos não praticantes, indicando que o treino físico por si só, é um colaborador importante na melhora do controle postural.

Brouwer et al. (2003) sugerem que indivíduos que apresentam reduzidos níveis de equilíbrio apresentam uma elevada preocupação com a ocorrência de quedas e pouca confiança no seu próprio equilíbrio, limitando por consequência as suas atividades. Ou seja, existe um ciclo vicioso onde a inatividade acentua a perda funcional determinante na manutenção do equilíbrio postural e, esta perda de equilíbrio acarreta em medo de cair, que por sua vez, restringe ainda mais a atividade cotidiana do idoso. Confirmando os achados da literatura, o presente estudo mostra que a hidrocinesioterapia realizada em piscina rasa ao influenciar positivamente 0 controle postural e equilíbrio pode ajudar a "quebrar" este ciclo, devolvendo a funcionalidade, 
a autonomia e a qualidade de vida aos idosos (LORD et al., 2003).

Dentre as limitações do estudo, pode ser citado o pequeno número de participantes da amostra e a inexistência de um grupo controle, em virtude da difícil paridade encontrada entre os indivíduos institucionalizados. Assim, sugere-se que em futuros estudos um número maior de indivíduos seja avaliado com testes capazes de isolar a contribuição de cada sistema sensorial, visando aperfeiçoar os tratamentos e terapias para melhorar o controle de postura dessa população.

\section{Conclusões}

De acordo com os resultados encontrados é possível inferir que a hidrocinesioterapia realizada em piscina rasa é capaz de influenciar os sistemas sensoriais responsáveis pelo controle da postura de mulheres idosas institucionalizadas. As principais diferenças após a aplicação da terapia foram encontradas no deslocamento do centro de pressão na direção ântero-posterior, que apresentou significativa diminuição de deslocamento na maioria das condições do TOS.

\section{Referências}

AMIRIDIS, I. G.; HATZITAKI, V.; ARABATZI, F. Age-induced modifications of static postural control in humans. Neuroscience Letters, Ireland, v.350, p.137-140, 2003. Disponível em: http://www.ncbi.nlm.nih.gov/pubmed/14550913. Acesso em: março 2011.

BALOH, R. W.; JACOBSON, K. M.; ENRIETTO, J. A.; CORONA, S. HONRUBIA, V. Balance disorders in older persons: Quantification with posturagraphy. Otolaryngology-Head and Neck Surgery, Alexandria, v.119, p. 89-92, 1992. Acesso em: outubro 2011.

BELLEW, J. W.; PANWITZ, B. L.; PETERSON, L.; BROCK, M. C.; OLSON, K. E.; STAPLES, W. $\mathrm{H}$. Effect of acute fatigue of the hip abductors on control of balance in young and older women.

Archives of Physical Medicine and Rehabilitation, Reston, v. 90, p. 1170-1175, 2009. Disponível em:

http://www.sciencedirect.com/science/article/pii/SO 003999309002706. Acesso em: janeiro 2011.

BIASOLI, M. C.; MACHADO, C. M. C.

Hidroterapia: técnicas e aplicabilidades nas disfunções reumatológicas. Temas de

Reumatologia Clínica, Rio de Janeiro, v.7, n.3, p.78-87, 2006. Disponível em:

http://www.biasolifisioterapia.com.br/publicacoes/T
Reuma3-06-Hidroterapia.pdf. Acesso em: outubro 2009.

BROUWER, B. J.; WALKER, C.; RYDAHL, S. J.; CULHAM, E. G. Reducing fear of falling in seniors through education and activity programs: a randomized trial. Journal of the American Geriatrics Society, Malden, v.51, n.6, p.829-834, 2003. Disponível em:

http://www.ncbi.nlm.nih.gov/pubmed/12757571. Acesso em: setembro 2010.

CAMPION, M. R. Hidroterapia - Princípios e Prática. Ed. Manole. São Paulo, SP, 2000.

CANDELORO, J. M.; CAROMANO, F. A. Efeito de um programa de hidroterapia na flexibilidade e na força muscular de idosas. Revista Brasileira de Fisioterapia, São Paulo, v.11, n.4, p.303-309, 2007. Disponível em:

http://www.scielo.br/pdf/rbfis/v11n4/a10v11n4.pdf. Acesso em: março 2011.

CARVALHO, J.; PINTO, J.; MOTA, J. Actividade física, equilíbrio e medo de cair. Um estudo em idosos institucionalizados. Revista Portuguesa de Ciência e Desporto. Porto, v.7, n.2, p.225223, 2007. Disponível em:

http://www.scielo.oces.mctes.pt/pdf/rpcd/v7n2/v7n 2a11.pdf. Acesso em: setembro 2010.

CASTAGNO, L. A. A new method for sensory organization tests: the foam-laser dynamic posturography. Revista Brasileira de Otorrinolaringologia, São Paulo, v.60, n.4, p.287-296, 1994. Disponível em: http://www.rborl.org.br/conteudo/acervo/print acer vo english.asp?id=2600. Acesso em: março 2012.

DANIEL, F.; VALE, R.; GIAN, T.; BACELLAR, S.; DANTAS, E. Effects of a Physical Activity Program on Static Balance and Functional Autonomy in Elderly Women. Macedonian Journal of Medical Sciences, Republic of Macedonia, v.15, n. 3, p. 16, 2010. Disponível em:

http://www.mims.ukim.edu.mk/Online/MJMS 201 03 1/MJMS.1857-5773.2010-0083.pdf. Acesso em: janeiro 2011.

DE VITO. C. A.; MORGAN, R. O.; DUQUE, M.; ABDEL-MOTY, E.; VIRNIG, B. A. Physical performance effects of low-intensity exercise among clinically defined high-risk elders.

Gerontology, Freiburg im Breisgau, v. 49, n. 3; p. 146-154, May/Jun, 2003. Disponível em: http://www.ncbi.nlm.nih.gov/pubmed/12679604. Acesso em: março 2010.

FERREIRA, F. P. M. Produção do Journal of biomechanics entre os anos 2000 e 2001 relacionado ao tema equilíbrio corporal, Rio de 
Janeiro - RJ, 2003. Disponível em:

http://www.pucrs.br/feng/microg/labs/nuba/monogr afias dissertacoes/monografias dissertacoes 01. pdf. Acesso em: outubro 2011.

GAUCHARD, G. C.; GANGLOFF, P.; JEANDEL, C.; PERRIN, P. P.; Physical activity improves gaze and posture control in the elderly. Neuroscience

Research, Japan, v.45, n.4, p.409-417, 2003.

Disponível em:

http://www.ncbi.nlm.nih.gov/pubmed/12657454.

Acesso em: setembro 2011.

GREVE, P.; GUERRA, A. G.; PORTELA, M. A.; PORTES, M. S.; REBELATTO, J. R. Correlações entre mobilidade e independência funcional em idosos institucionalizados e não-

institucionalisados, Fisioterapia em Movimento, Curitiba, v. 20, n.4, p.117-124, 2007. Disponível em:

http://www2.pucpr.br/reol/index.php/RFM?dd1=17 83\&dd99=view. Acesso em: agosto 2011.

HENRY, C.; WEBSTER-GANDY, J.; VARAKAMIN, C. A comparison of physical activity levels in two contrasting elderly populations in Thailand. American Journal of Human Biology, Malden, v. 13, p.310, 2001. Disponível em: http://www.ncbi.nlm.nih.gov/pubmed/11460896. Acesso em: outubro 2011.

LIU-AMBROSE, T.; KHAN, K. M.; JANICE, J. Resistance and Agility Training Reduce Fall Risk in Women Aged 75 to 85 with Low Boné Mass: A 6-Month Randomized Controlled Trial, Journal of American Geriartric Society, Malden, v.52, p.657-665, 2004.

LORD, S. R.; CASTELL, S.; CORCORAN, J.; DAYHEW, J.; MATTERS, B.; WILLIAMS, P. The effect of group exercise on physical functioning and falls in frail older people living in retirement villages: a randomized, controlled trial. Journal American Geriatric Society, Malden, v.51, n.12, p.1685-1692, 2003. Disponível em: http://www.ncbi.nlm.nih.gov/pubmed/14687345. Acesso em: setembro 2011.

MOCHIZUKI, L.; DUARTE, M.; AMADIO, A. C.; ZATSIORSKY, V. M.; LATASH, M. L. Changes in Postural Sway and Its Fractions in Conditions of Postural Instability. Journal Applied

Biomechanics, Providence, v.22, p.51-60, 2006. Disponível em:

http://www.demotu.org/pubs/JAB06.pdf. Acesso em: outubro 2011.

MOCHIZUKI, L.; AMADIO, A. C. Aspectos biomecânicos da postura ereta: a relação entre 0 centro de massa e o centro de pressão. Revista Portuguesa de Ciência e Desporto, Porto, v.3, n.3, p.77-83, 2003. Disponível em: http://www.fade.up.pt/rpcd/ arquivo/artigos soltos /vol.3 nr.3/Mochizuki.pdf. Acesso em: agosto 2011.

RAY, C. T.; HORVAT, M.; CROCE, R.; MASON, R. C.; WOLF, S. L. The impact of vision loss on postural stability and balance strategies in individuals with profound vision loss. Gait and Posture, Oxford, v.28, p. 58-61, 2008. Disponível em:

http://www.ncbi.nlm.nih.gov/pubmed/18023185. Acesso em: janeiro 2012.

RAYMAKERS JA, SAMSON M.M, VERHAAR H.J.J. The assessment of body sway and the choice of the stability parameter(s). Gait and Posture, Oxford, v.21, p.48-58, 2005. Disponível em:

http://www.ncbi.nlm.nih.gov/pubmed/15536033. Acesso em: outubro 2011.

RESENDE, S. M.; RASSI, C. M.; VIANA, F. P. Efeitos da hidroterapia na recuperação do equilíbrio e prevenção de quedas em idosas. Revista Brasileira de Fisioterapia, São Carlos, v.12, n.1, p.57-63, 2008. Disponível em: http://www.scielo.br/pdf/rbfis/v12n1/11.pdf. Acesso em: janeiro 2012.

RICCI, N. A.; GAZZOLA, J. M.; COIMBRA, I. B. Sistemas sensoriais no equilíbrio corporal de idosos. Arquivos Brasileiros de Ciência e Saúde, Santo André, v.34, n.2, p.94-100, 2009. Disponível em: http://files.bvs.br/upload/S/19832451/2009/v34n2/a006.pdf. Acesso em: dezembro 2011.

RONDA, J. M.; GALVAÑ, B.; MONERRIS, E.; BALLESTER, F. Associacion entre sintomas clínicos y resultados de la posturografia computadorizada dinámica. Acta

Otorrinolanigológica Española. Madrid, n.53, p.252-255, 2002. Disponível em: http://www.elsevier.es/sites/default/files/elsevier/p df/102/102v53n4a13097886pdf001.pdf. Acesso em: setembro 2011.

STEL, V.S., SMIT, J.H., PLUIJM, S.M.F., LIPS, P. Balance and mobility performance as treatable risk factors for recurrent falling in older persons. Journal Clinical Epidemiological, Ottawa, v. 56, p.659-668, 2003. Disponível em:

http://www.eng.auburn.edu/ie/ose/jc\%20articles/fal 1 predictors.pdf. Acesso em: agosto 2011.

TEIXEIRA, C. S, DORNELES, P. P, LEMOS, L. F. C.; PRANKE, G. I.; ROSSI, A. G.; MOTA, C. B. Avaliação da influência dos estímulos sensoriais envolvidos na manutenção do equilíbrio corporal em mulheres idosas. Revista Brasileira de Geriatria e Gerontologia, Rio de Janeiro, v.14, n.3, p.453-460, 2011. Disponível em: 
http://revista.unati.ueri.br/pdf/rbgg/v14n3/v14n3a0

6.pdf. Acesso em: outubro 2011.

WOLFSON, L.; WHIPPLE, R.; DERBY, C. A.;

AMERMAN, P.; MURPHY, T.; TOBIN, N.;

NASHNER, L. A dynamic posturography study of

balance in healthy elderly. Neurology,

Minneapolis, v.42, n.11, p.2069-2075, 1992.

\section{Endereço:}

Estele Caroline Welter Meereis

Departamento de Fisioterapia e Reabilitação,

Centro de Ciências da Saúde - UFSM

Av. Roraima, 1000 Bairro: Camobi

Santa Maria RS Brasil

97105-900

Telefone: (55) 81281728

e-mail: estelemeereis@gmail.com

Recebido em: 01 de abril de 2012.

Aceito em: 29 de março de 2013.

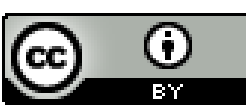

Motriz. Revista de Educação Física. UNESP, Rio Claro,

SP, Brasil - elSSN: 1980-6574 - está licenciada sob Creative Commons - Atribuição 3.0 